\title{
Analyzing the Effects of Role Configuration in Logistics Processes using Multiagent-Based Simulation: An Interdisciplinary Approach
}

\author{
Ingo J. Timm, Lukas Reuter, Jan Ole Berndt \\ Center for Informatics Research and Technology \\ Trier University \\ $\{$ itimm, reuter, berndt $\} @$ uni-trier.de
}

\begin{abstract}
Current trends like the digital transformation and Industry 4.0 are challenging logistics management: flexible process development and optimization has been a primary concern in research in the last two decades. However, flexibility is limited by its underlying distribution of action and task knowledge.

Thus, our objective is to develop an approach to optimize performance of logistics processes by dynamic (re-) configuration of knowledge in teams. One of the key assumptions for that approach is, that the distribution of knowledge has impact on team's performance. Consequently, we propose a formal specification for representing active resources (humans or smart machines) and distribution of action knowledge in multiagent-based simulation. In the second part of this paper, we analyze process quality in a psychologically validated laboratory case study. Our simulation results support our assumption, i.e., the results show that there is significant influence of knowledge distribution on process quality.
\end{abstract}

\section{Introduction}

Logistics and process optimization is an important field of research in theory and practice. Various approaches on coordination and implementation of information exchange as well as process and workflow execution by information systems have been developed as part of enterprise resource planning and logistics management systems. To increase efficiency and effectivity of production in a globalized world, innovative approaches propose decentralization and autonomy $[1,2,3]$. Thus, information exchange, workflow specification and implementation of workflows by information systems has been in focus of early approaches to enterprise resource planning and logistics management systems.

However, these approaches mainly focus on emergent coordination, organization, and

\author{
Anna-Sophie Ulfert, Thomas Ellwart, Conny Antoni \\ Business, Work and Organizational Psychology \\ Trier University \\ \{ulfert, ellwart, antoni\}@uni-trier.de
}

(decentralized) planning of logistics processes. In practice flexible behavior of resources, e.g., workers or smart machines, heavily depend on its skills and capabilities. A resource can only be used in process steps, if its skills and capabilities match the requirements of the process. If resource capabilities are only partially matching task requirements, there will be reduced quality or efficiency of the process execution. Skills and capabilities are part of the action and task knowledge of the resources in logistics and are typically heterogeneous between the individual resources within in a system. Human resources improve their action and task knowledge by application of the corresponding action or task as well as by training and learning. If task knowledge and action is not actively applied, it will fade out, i.e., it will be forgotten, such that the level of specialization of a resource decreases. In organizational design, roles are defined for binding actions and tasks to resources or groups of resources. Binding many roles to one resource increases their general applicability but restricts its ability to improve on the skill and task limits due to (cognitive) capacity restrictions. Resources only connected to a focused action and task area will increase the specialization of this resource while flexibility, e.g., serving as a substitute for a different role, will be restricted. From an organizational perspective the (re-) allocation of roles to resources is a process where new knowledge has to be learned, e.g., adding new roles, or knowledge should be intentionally forgotten, e.g., when roles are deleted from a resource.

Planning and adapting the role model, i.e., the knowledge distribution of the organization, is part of organizational development and evolution on basis of psychological and economical considerations [4]. In a digitalized world where environmental and internal changes appear on short-term, adaptations to roles at design time of the processes are not sufficient to ensure reliable and flexible processes. So we propose an interdisciplinary approach to dynamic role assignment by intentional forgetting, such that the configuration of knowledge in the organization, i.e., 
the distribution of specialized and general purpose resources is balanced. Based on theories and empirical findings from psychology on human team cognition as well as deliberative agent theories from distributed artificial intelligence (DAI) [5], we are developing an interdisciplinary model on intentional forgetting for dynamic role allocation in teams. This research is based on the key assumption, that the distribution of knowledge has impact on team's performance, i.e., that efficiency and quality of processes are depending on the role configuration. In psychological theory, the dimensions of knowledge distributions in teams are shared and distributed knowledge among team members. In a fully distributed, i.e., partitioned, knowledge structure each team member is specialized on a particular area of expertise and in a completely shared knowledge structure each team member as a generalist is able to processes every kind of tasks. The probability of errors in the process increase, if certain employees are overloaded by work or information, e.g., due to a too broad, reps. generalist knowledge structure. To cope with overload in work scenarios, knowledge structures can be reconfigured by intentional forgetting and focusing on fewer amount of roles per resource.

As the decision on the optimal knowledge distribution in teams is situation-dependent, there is no silver bullet in design time of the processes. Dynamic analysis of current situations are required, such that simulation seems to be a promising approach to support dynamic reallocation of roles. Simulation as an instrument can be used to predict different scenarios and analyze complex dependencies within the teams. Therefore, we propose the application of multiagent-based simulation (MABS) as it is capable of representing human's and smart machine's decision making as well as knowledge base. Within this paper, we introduce a formal specification of the required models for resources (agents) and teams (multiagent system) allowing representation of action and task knowledge distribution. We analyze our key assumption - the influence of knowledge distribution to process quality and efficiency - using this specification in context of a psychological experiment.

The contribution of this paper is an interdisciplinary concept for modeling knowledge distribution in teams as well as measuring performance effects. In order to simulate the effects, a rigorously formalized specification of the model based on DAI theories is used to represent human knowledge distribution and decision-making in intelligent agents. The question of performance advantages through different knowledge structures is shown in context of a laboratory case study which is conducted using a serious game (networked fire chief). It allows for representing complex group decisions in a controllable environment.

The remainder of this paper is structured as follows. Section 2 provides insights of autonomous and decentralized processes management in logistics as well as theoretical foundations for the conceptual model. Section 3 gives an overview of psychological foundations of knowledge configurations in teams. The interdisciplinary knowledge (re-)configuration model for MABS is presented in Section 4. A case-study to evaluate potential knowledge configuration in teams and measure their effects is conducted and described in Section 5 .

\section{Autonomous Logistics}

In contrast to conventional approaches in logistics planning and scheduling by means of operations research, autonomous logistics focuses on the representation of active elements in logistics processes [6]. Doing so, decentralized decision-making is implemented which on the one side seems to be an adequate mean for modern production and logistics infrastructure in context of mobile and ubitiquous computing. On the other hand, by decentralization, the computational complexity is decreased significantly and realtime planning and reacting to changes is enabled. For example, in logistics, such an approach can decrease the computational effort for solving a distributed vehicle routing problem [7]. In this case, local groups of cooperating entities constrain the number of parameters to take into account for decomposing the overall task into computationally tractable sub-problems.

From a conceptual perspective, there are two main approaches to implementing autonomous logistics: as a bottom-up approach using integrating techniques like Internet-of-Things and service-oriented computing and as a top-down approach using multiagent systems. For our research, multiagent systems are of concern, as the knowledge representation and flexible organization is too limited in the other case. Software agents as intelligent representatives of real-world objects and facilities incorporate the state of their corresponding physical entities. It is assumed, that these agents take control of their functions and receptions, e.g., an agent is aware of information available to the physical entity and has knowledge on its capabilities of accepting transport orders for its available services. Depending on the software architecture of an agent, it has individual objectives and explicit planning capabilities [8].

With respect to the horizontal and vertical integrative function of logistics, agents have to coordinate their 
activities with each other or with external processes. While, negotiation, e.g., on price or time, does not necessarily require agent technology and could also be implemented as web-services, flexibility can significantly benefit from goal-driven coordination of intelligent agent, e.g., [9]. Following such approaches, multiagent systems are dynamically agree on their dependencies, such that the organization of the multiagent system emerges by their interaction behavior [10]. In context of our research, these interaction behavior is part of process execution and not changing the organizational structure by re-allocating capabilities or roles. Due to the self-similarity of logistics objects from on to another level of abstraction, e.g., package, shipment, container, there has been various approaches to bind agents together as a result of coordination and continue planing, coordination, and control by one representative of the complete group of bounded agents. These concepts refer to holons or holonic systems, inspired by social theories [11]. For instance, in manufacturing and production planning, so-called holonic organizations have been used for the joint planning and execution of tasks [12, 13, 14]. While combining knowledge bases is discussed in context of holon research, holons tend to be task oriented, so that they come closer to short-term organization of process execution rather than fundamental support of role assignment. Additionally, the property of self-similarity aims at integrating similar elements together rather than constructing dynamic knowledge structure in an organization.

Situation-dependent adaptation of groups or organization is primarily a sociological phenomena as well as handled by business administration. Various approaches exist, transferring theories and methodologies from those disciplines to multiagent research, e.g., [15, 16]. Social mechanisms have been researched for their application in logistics for dynamic (re-) organization of interdependent actors, eg., $[17,18,19]$. However, these approaches are rather focused on interaction patterns than on knowledge explicitly. Knowledge representation and processing explicit knowledge are important aspects of multiagent systems research. In logistics, the majority of the approaches deal with interaction design as coordination of activities is one of the main tasks, here. In the beginning of multiagent systems, there have been multiple approaches to adopting intelligent agents, e.g., $[20,21]$. The practical reception of those approaches is limited and Davidsson et al. propose that more validation is required first [22]. Thus, these approaches provide a formal basis for specification of multiagent systems but the resulting models have to be validated.
Finally, learning is a strategy for adapting knowledge within agents and, doing so, implicitly rearrange roles and knowledge within the multiagent system. There is a broad range of learning approaches reaching from abstraction and aggregation of data, connectionists approaches like Neural Networks, reinforcement learning or inductive logic programming, e.g., [23, 24, 25, 19]. Approaches of agent-based learning in logistics can be applied for implementing the adaptation of agents to new situations or to acquire experience. However, for the specification of our interdisciplinary model, intentional forgetting as an inverse function to learning as well as learning and intentional forgetting on an organizational, i.e., team, layer is required.

\section{Psychological Teamwork Foundations on Roles and Processes}

In order to analyze, model and design roles and processes in organizations and measure their performance, insights from psychology are helpful to understand team functioning and cognition. In psychological group-research, teams can be defined as collective information-processing systems [26]. Team members memorize knowledge required for their tasks, they specialize on particular areas of expertise, or they share knowledge and information with each other [27, 28]. These various approaches to the organization of team knowledge are known as team cognitions [29]. Team cognitions describe the structure in which knowledge important to team functioning is mentally organized, represented, and distributed within the team and allows team member to anticipate and execute actions [30]. Therefore, they are particularly suitable as a theoretical concept for describing, modeling, and analyzing knowledge (re-)configuration approaches in collaborative work processes. Team cognition, as an emergent state, are conceptualized as (1) shared team knowledge or (2) distributed team knowledge [30].

When working together, it is important for team members to share their knowledge about task and team relevant information with each other in the form of team mental models to facilitate successful cooperation and coordination [30]. On the one hand, this generates trust and increases coordination and the robustness of the work process against disturbances by means of information exchange and the acquisition of group knowledge [31, 32]. On the other hand, sharing of the entire knowledge among all team members results in an increased amount of information that needs to be processed by each individual which can lead to information overload [33, 34]. Information overload endangers the effectiveness and efficiency of the team 
as its members struggle to focus on current tasks when constantly switching between different contexts [30].

Contrastingly, specializing on particular areas of competence reduces the cognitive load faced by members of a team [35]. That is, each team member can focus on her specific expertise which reduces the load of information being processed. This distribution of knowledge in specialized teams increases the overall knowledge capacity of the whole team since individual members only have to memorize and process information which is relevant to their areas of expertise [36]. However, this potentially makes the team as a system more fragile as it lacks the required redundancy of knowledge to avoid confusion, conflicts, and failures [37].

In fact, a completely specialized team will be dysfunctional. This is because effective coordination between its members requires them to have some meta-knowledge about their co-workers' competencies and the overall process of collaboration. Such a transactive memory provides a shared context for the performance of divided tasks [28]. It contains knowledge about the location of expertise (knowing who knows what) which facilitates cooperation, coordination, and further specialization [36]. Moreover, a transactive memory even increases both a team's performance in dynamic environments and its ability to include new members [38, 39].

If certain information and knowledge is not equally memorized by all team members then the overall system capacity is increased [36]. Consequently, particular information and knowledge can be ignored by other team members. Therefore, we define intentional forgetting as a deliberate reconfiguration of knowledge distributions in teams and organizations to extend their knowledge capacity. A MABS combining psychological grounded representation of human teamwork decision-making and performance with formalization and modeling from distributed artificial intelligence allows for comprehensive simulation, analysis and understanding of modern production processes. By means of an intentional forgetting mechanism, roles and processes can be reconfigured and optimized.

\section{Modeling and Configuring Knowledge Distribution in Multiagent-Teams}

In order to apply the mechanism of intentional forgetting to roles and processes in organizations, this Section presents an interdisciplinary approach of theories from psychology and formalization from DAI. In Figure 1, the overall intentional forgetting model of knowledge distribution, measuring effects and performance of knowledge distributions and reorganizing knowledge is shown. The next paragraphs show a detailed formalization of the model components. In Teams, each team member has specific capabilities

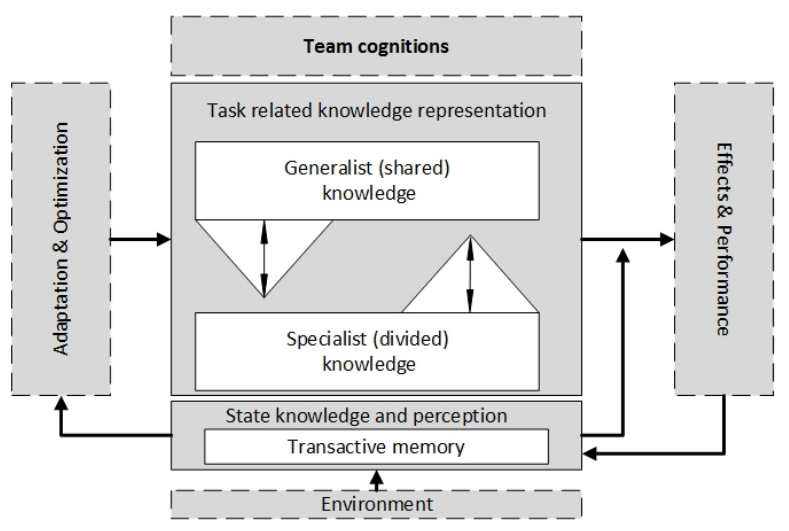

Figure 1. Modeling, Measuring and Adapting Knowledge Distributions in Teams

which represent task related knowledge. According to psychological group research, the dimensions of capability distributions among team member can be either totally distributed or completely shared and both are coming with their advantages (see Section 3). In order to apply a mechanism of intentional forgetting to agents, the formalization of an agent and its capabilities is needed. The following formalization of an agent $A g$ is based on logics and concepts from [40, 41, 42]. Moreover, the actual definitions are derived from $[20,1]$.

The see and execute functions of an agent are interfaces to its environment (see Definition 4.3). The intentional forgetting mechanism, i.e., a shift in the capability configuration of an agent, is implemented in an agent's reflect function. It allows for a reconfiguration of an agents local state.

Definition 4.1. An agent is given by a 7-tuple: $A g=$ $\left\langle L\right.$, Act, see, reflect, decide, execute, $\left.l_{0}\right\rangle$ where:

- $L$ as a representation of the local state of the agent, see Definition 4.2 for a detailed formalization

- Act is a set of Actions an agent can perform

- see is a perception function which maps an environment to an agent's perception see : Env $\rightarrow$ Perc

- reflect is a function to update $L$; reflect : $L \times$ $\operatorname{Perc} \rightarrow L^{*}$

- decide is a function which transforms the local state to action plan 
- execute is a function which selects a single action from an action plan and executes it; execute : $L \rightarrow A c t$

- $l_{0} \in L$ as initial internal agent state

Besides actions concerning environmental changes of an agent, a communication interface is modeled. Hence, the knowledge on actions of an agent is given by $A c t$ and can be divided in $A c t_{e}$ for actions in the environment and $A c t_{c}$ for communicative actions such as message sending or receiving. In this approach, the knowledge on action is considered as being static during runtime of the system, i.e., the agent does not include a function to reflect on isolated actions. The role specification for an agent consists of knowledge on the objectives (what it has to do in this role) and on the procedures (how should a task be accomplished following this rule). This knowledge is part of the dynamic knowledge base of the agent, i.e., its local state. The local state embraces the agent's beliefs about the perceived environmental information as well as the reflection of its own condition. As task knowledge, the desires are directing the agents behavior as a representation of persistent goals. The procedural knowledge for tasks is stored as plan templates which can also include specific planning and execution routines. The local state of an agent is defined as follows:

Definition 4.2. An agent's local state $L$ is given by a 5-tuple: $L=\langle B, D, I, P \ln , \gamma\rangle$ where:

- $B \subset B^{*}$ is the set of current beliefs

- $D \subset D^{*}$ is the set of current desires

- $I \subset D \times P l n$ is the set of active intentions,

- Plan $\subset P l n$ is the set of available plans, and

- $\gamma: B * \times D \rightarrow \mathbb{R}$ is a mapping computing the relevance of a desire in the current situation.

To be more exact, the capabilities of an agent are implicitly modeled in its plan base. In order to solve particular tasks an agent can execute a given plan, i.e., a chain of actions to process it. In order to visualize the agent function and its dependencies, a schematic agent architecture is shown in Figure 2. In order to allow for a team-based intentional forgetting comprehension, the definition of agent teams, i.e., multiagent system is necessary. But beforehand a definition of an agent environment is needed.

Definition 4.3. An agent environment is defined by: $E n v=\left\langle E, A_{c t}, \ldots, A c t_{n}, \tau, e_{0}\right\rangle$ where:

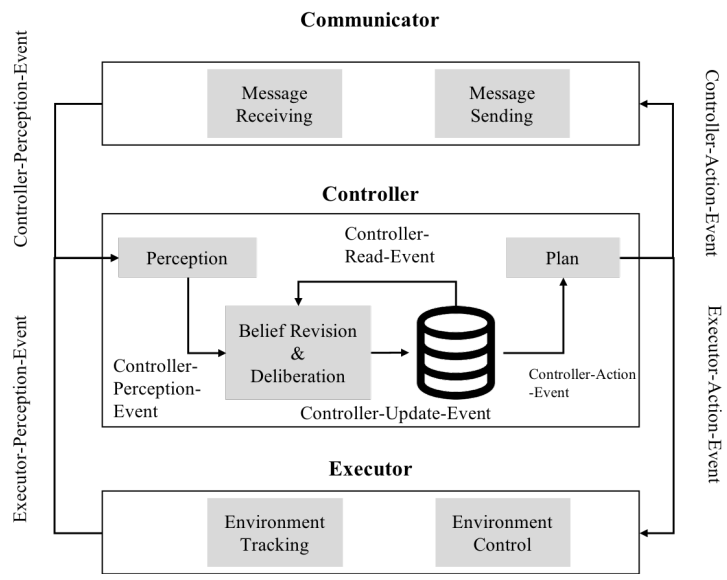

Figure 2. Schematic Modular Agent Architecture

- $E$ is the set of all possible environmental states,

- $A c t_{i}$ is the set of actions for each Agent $A g_{i}$ with $i=1, \ldots, n$,

- $\tau: E \times A c t_{1} \times \ldots \times A c t_{n} \rightarrow E$ is defining a state transformation function,

Consequently, with the use of an agent environment, a MAS can be formalized as follows.

Definition 4.4. A Multiagent system, i.e., a Team is defined as: $M A S=\left\langle E n v, A g_{1}, \ldots, A g_{n}\right\rangle$ where:

- Env is an agent environment as defined in Definition 4.3

- and a sequence of agents $A g_{i}$ as defined in Definition 4.1 with $i=1, \ldots, n$

Having a formalized MAS it is feasible to define capability configurations for agent teams.

Definition 4.5. The capabilities of a team $T C$ is the union of the capabilities, i.e., the plans of every agent: $T C=\cup_{A g \in M A S} P l n_{A g}$ The dimensions of generalist and specialist knowledge distributions are defined as:

- Shared agent capabilities: $T C=P \ln _{A g_{1}}=\ldots=P \ln _{A g_{1}}$. Every agent has the same amount of capabilities and therefore allowed to process all kind of tasks.

- Distributed agent capabilities: $T C=$ $\exists \dot{U}_{A g \in M A S} P \ln _{A g}$ For any kind of tasks, there is a specialist who is only capable of processing it.

In order to achieve effective and efficient teamwork, a suitable capability structure is needed. Each team configuration is accompanied by individual performance 
and robustness properties. Regarding the hypothesis of performance increase, specialist teams are processing tasks more efficient than generalists due to a lower cognitive load. To decide which capability configuration is suitable for a specific situation, the definition of performance indicators is necessary.

Definition 4.6. A performance indicator $P I$ for MAS in an organizational context can be defined as: $P I$ : $E \rightarrow \mathbb{R}$ with an environmental state $E$ mapped onto an evaluation space of rational numbers.

In production processes, the effectiveness is mostly measured by time-based key performance indicators such as the troughput time [43]. Due to psychological research, quality should be considered when evaluating knowledge capacity. Therefore, we specify the error frequency as an indicator for cognitive overload of team members which indicates process quality (cf. six sigma [44]). If team member have different competences and responsibilities among various work contexts, it is possible that they lead to a state of information overload. This may cause higher error rates in work processes and a lack of work quality. The next section provides an exemplary model and PI implementation to test impacts of capability distributions among human teams in a controllable environment and transfer the observations to agents.

\section{Case-Study: Effects of Roles and Process Configurations}

In order to test the hypothesis of performance advantages of specialist knowledge structures, i.e., specialized agent capabilities a case study is conducted. The aim of this case study is to analyze a team's performance for different knowledge structures and their ability to cope effects of increasing task complexity, e.g., information overload and to transfer results from human teams to multiagent teams. In order to show these effects a serious game, the networked fire chief (NFC) is chosen. The NFC platform allows for data collection in small group research as well as a structured environment for agent interaction. NFC is designed for enabling complex decision-making with controllable conditions [45]. The combination of laboratory experiments and MAS, is a promising approach to allow for human decision-making in agents [46, 47]. By reallocation and goal-directed usage of given resources, NFC addresses core challenges of modern logistic processes and is therefore appropriate environment for analyzing performance effects of varying role configurations.

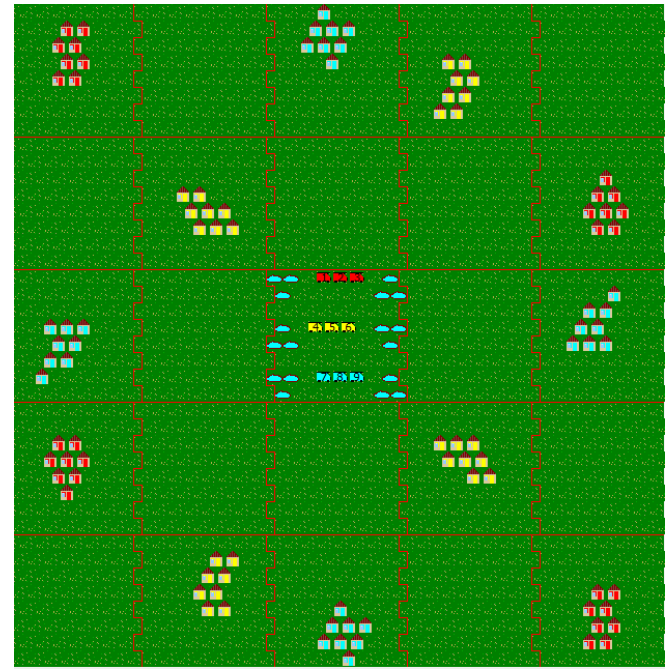

Figure 3. Networked Fire Chief Scenario Setup

\subsection{Scenario Specification}

In the specified scenario, a team of three (humans as well as agents) is working together to extinguish fire. This environment is structured in a $40 \times 40$ array which consists of houses, grassland, firetrucks and lakes (see Figure 3). The environment is controlled by three fire stations which individually controlled by a participant. Each station is responsible for a set of firetrucks. In order to measure the effects of sharing and dividing knowledge,i.e., capabilities, rules for extinguishing fire as well as refilling firetrucks are defined using relations between the entities firetruck $F T$, house $H$ and lake $L$. A rule $R$ is a combination of valid usage of colored entities. Hence, a rule for a participant with specialized capabilities on red entities for extinguishing fire is a mapping of: $R_{1}: F T_{\text {red }} \rightarrow H_{\text {red }}$ as well as for refilling: $R_{2}: F T_{\text {red }} \rightarrow L_{\text {blue }}$. These rules describe that this station is allowed to control all red firetrucks, to extinguish fire on red houses, and to refuel with water from blue lakes. Other team member are specialized on the remaining colors yellow and blue. Such a setup represents a fully divided knowledge configuration. For generalist knowledge structure, each station is allowed to control one firetruck of each color with corresponding coloring restrictions. This represents a maximum on sharing knowledge.

In order to test the performance differences, the scenario is divided into three different complexity classes. In more complex scenarios the color combinations of the entities are not harmonic, e.g., red firetrucks can extinguish fire in green houses and refuel on purple lakes. In experiments is proven that, for example, humans are less efficient naming colors 
of words when they are written in a different color (Stroop Effect [48]). Hence, using multiple color combinations to model task complexity is a suitable approach. For each complexity class, the combination of color matching entities is increasing. In order to measure performance, failure indicators for using the wrong kind of firetruck, refill at a wrong lake or extinguish the wrong colored house.

\subsection{Agent Behavior}

Each agent represents a test subject in the simulation which controls a fire station. Based on the agent formalization model in Section 4, the agent is separated in decision-making (controller) and executing decisions (executor) to provide a fully modular and reusable agent architecture (Figure 2). However, following the process for the laboratory setup there is no communication between the test subjects intended. Therefore the agent interactions are limited to the environment.

In the implemented agent model, the executor provides an interface for a simulation framework and signals environmental changes as well as executes actions. In this approach the Repast Simphony simulation framework is used. The controller is also fully modular and consists of four components: Perception, Decision-Making, Knowledge-Base and Action. Each component is event-driven, that is, each component can fire and handle events which allows for model extensions in future simulations. The decision-making itself is rule-based,i.e., plan-based according to the rules each fire station has. Therefore, an agent can execute two different plan-categories, extinguish fire and water refilling. That is, for each rule in the scenario there is a separate and invariant plan.

An agent chooses refilling if there is not enough water to extinguish a fire. It then selects the nearest refilling opportunity. In order to choose a fire to extinguish, each agent has a representation of fire locations and selects the nearest one. It chooses burning houses over burning landscapes. If there is another firetruck which is also allowed to extinguish this kind of fire and is actually nearer to the fire, then the agent chooses the next fire.

Based on the plans given, an agent would not make mistakes such as choosing the wrong kind of houses, firetruck or lake. However, human test subjects in the study do wrong choices. In order to transfer human decision-making to the agent's decision-making, each time an agent chooses an action a deviation parameter $\sigma$ for each selection is used. It is modeled as a threshold value to enable making wrong choices with a certain probability. Therefore three different deviation parameter $\sigma_{F T}$ : Probability of selecting correct Firetruck, $\sigma_{L}$ : Probability of selecting correct Lakes and $\sigma_{H}$ : Probability of selecting correct Houses, are used. The model is based on the assumption that the main decision-making is dependent on which firetruck is used. Each deviation parameter models the situation awareness of an agent concerning different entities [49]. For example the parameter $\sigma_{F T}$, in complex scenarios this parameter value can be lowered allowing more mistakes, e.g., a specialist on red entities is using a blue firetruck. The different $\sigma$ parameter are used to calibrate the simulation.

\subsection{Simulation and Experiment Results}

The result discussion is twofold, the first segments are focusing on the results of the laboratory experiment and the segments afterwards are showing the simulation results as well as their comparison to the experiment. As a performance indicator to evaluate a team's efficiency, the ratio of false actions to all actions is chosen. The calculation is based on moving the wrong kind of firetruck, extinguishing fire on a house with a wrong color and refilling water from a wrong lake according to the rules given. The main hypothesis for this experiment is: In complex work environments specialist knowledge structures will perform more efficiently than generalist knowledge structure according to failure indicators.

In Table 1 the average failure measurements from the experiment are shown. The experiment is conducted with 12 participants resulting in 144 NFC runs.

Table 1. Laboratory Experiment Results: average False Action Ratio

\begin{tabular}{lccc}
\hline Complexity & Low & Medium & High \\
\hline Specialist & 0,1544 & 0,1672 & 0,415 \\
\hline Generalist & 0,23 & 0,315 & 0,442 \\
\hline
\end{tabular}

In order to verify the hypothesis a two sided t-test was performed. Each series of measurement contains eight data points which represents the average of all false actions of each three person team. Figure 4 shows the individual test results for specialist and generalist knowledge structures in three different task complexity classes. For experiments with low and high complexity the results were not significantly distinct. In case of the second complexity class the results are significant with a p-value of 0.05 . It can be assumed that the scenarios with the highest complexity were too demanding and caused a state of information overload for both knowledge structures. Therefore hypothesis can be assumed for complexity class two.

The calibration of the agent-based simulation model is based on a sensitivity analysis of the parameter 


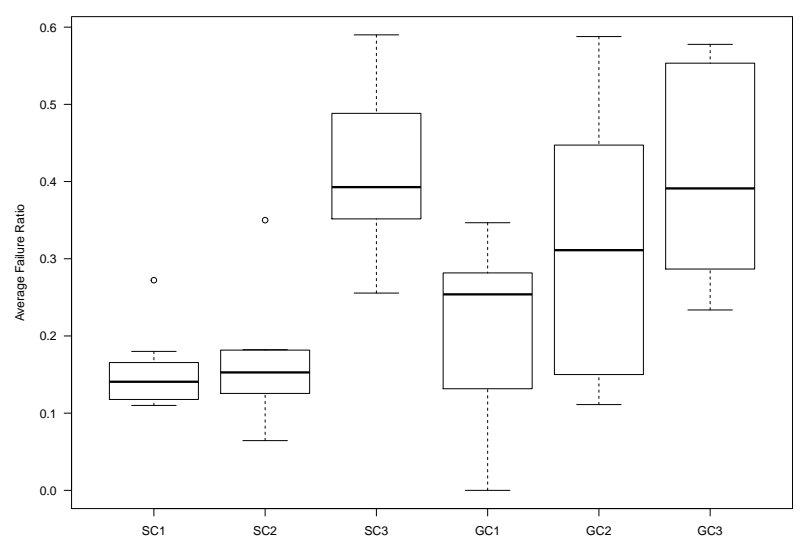

Figure 4. False Action Ratio from Laboratory Experiment

$\sigma_{F T}, \sigma_{H}$ and $\sigma_{S}$. Each parameter is varied in a interval $[0.0 ; 1.0]$ in steps of 0.1 , to get an overview of the parameter space. This analysis is performed with specialist and generalist knowledge structures and for each scenario complexity. Based on the results captured from the sensitivity analysis a simulation calibration is conducted. The results are shown in Figure 5. For each knowledge distribution and complexity degree the simulation was conducted 100 times.

The results of the simulation runs show similar characteristics concerning the failure behavior. However, the failure values in the simulation are more equally distributed among the complexity degrees. The distribution of the standard deviation in experiment and simulation is shown in Figure 6. The sample abbreviations are meant to read as: E,S for experiment or simulation; G,S for generalists or specialist and $\mathrm{C} 1$, C2, C3 for different complexity classes.

To show a more detailed behavior comparison of the simulation and experiment results, a single parameter setting is discussed. For example with a parameter setting of $\sigma_{H}=0,75, \sigma_{L}=0,44, \sigma_{F T}=0,745$ in a specialist knowledge structure and highest complexity class, the output is also similar in the simulation and the experiment (experiment: $\mu_{F T}=0,513, \mu_{L}=0,44$, and $\mu_{H}=0,28 /$ simulation: $\mu_{F T}=0,54, \mu_{L}=0,413$, and $\left.\mu_{H}=0,264\right)$. Concerning the average failure ratios the simulation produces similar outputs. However, the standard deviation of the experiment results for each single failure ratio parameter is very high $( \pm 0,65)$. This may arise from the small sample size in the experiment. In total, the MABS model is a suitable approach to replicate failure behavior in the NFC environment. According to average error parameter the laboratory

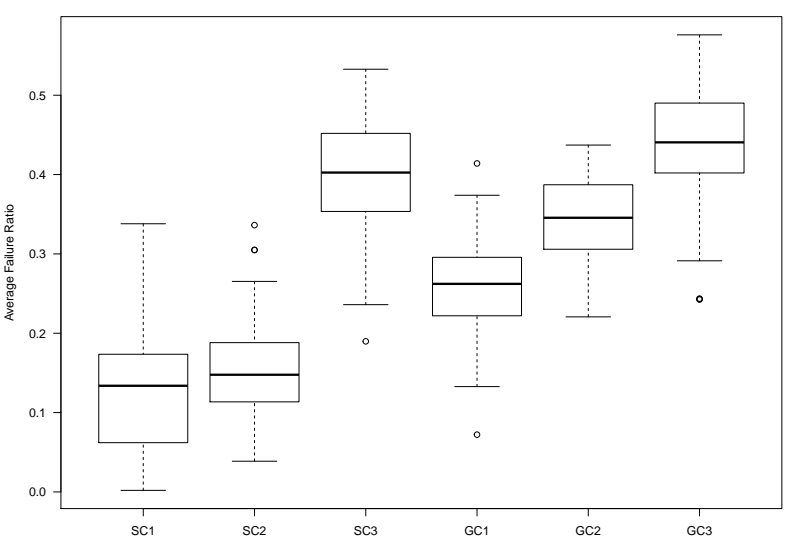

Figure 5. False Action Ratio for Simulated Knowledge distribution

experiment is being replicated by the simulation.

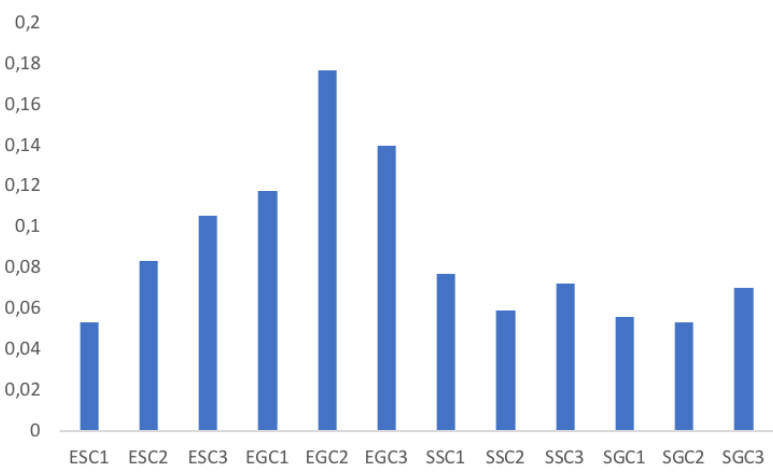

Figure 6. Standard Deviation Experiment and Simulation

\section{Conclusions}

The configuration of roles and processes in organization is a difficult task which needs a comprehensive interdisciplinary view. We have shown that the key assumption of our research, the distribution of knowledge has impact on a team's performance, can be verified. This paper proposes a simulation-based approach combining MABS and theories from psychology to configure knowledge distributions in teams by providing a formal agent model. The model allows for rigorous formulation of explicit task processing capabilities. From psychological theory it is known that specializing on certain expertise can enhance the cognitive capability which results in reducing errors in work processes. By conducting a laboratory experiment could be shown 
that specializing on particular areas of expertise team member does enhance process quality by reducing errors in process execution. Using a serious game environment revealed that teams with specialized task processing capabilities make significantly less errors in solving distributed tasks than generalist teams. Moreover, it could be shown that these results can be transferred to the multiagent model which enables realistic simulation of human knowledge capacity concerning error replication in processes. Such an agent model provides first insights in human cognition and can be seen as basis for reconfiguration, i.e., optimization roles and processes in organizational scenarios.

In future work this approach need to be extended to allow for dynamic reorganization of capability distributions among teams. This requires extensions on the agents' communication and reasoning abilities. By proving positive effects of intentional forgetting, dynamic capability management which allows for coping external and internal disturbances can be facilitated.

\section{Acknowledgments}

The project AdaptPRO: Adaptive Process and Role design in Organisations (TI 548/-1) is funded by the German Research Foundation (DFG) within the Priority Program "Intentional Forgetting in Organisations" (SPP 1921).

\section{References}

[1] I. J. Timm, P. Knirsch, H.-J. Kreowski, and A. Timm-Giel, "Autonomy in software systems," in Understanding Autonomous Cooperation and Control in Logistics, pp. 255-273, Springer, 2007.

[2] J. O. Berndt and O. Herzog, "Efficient multiagent coordination in dynamic environments," in IEEE/WIC/ACM International Conferences on Web Intelligence and Intelligent Agent Technology (WI-IAT) (O. Boissier, J. Bradshaw, L. Cao, K. Fischer, and M.-S. Hacid, eds.), (Lyon), pp. 188-195, IEEE Computer Society, 2011.

[3] A. Schuldt, Multiagent Coordination Enabling Autonomous Logistics. Berlin, Germany: Springer, 2011.

[4] I. J. Timm, J. O. Berndt, L. Reuter, T. Ellwart, C. Antoni, and A.-S. Ulfert, "Towards multiagent-based simulation of knowledge management in teams.," in Flexible knowledge practices and the Digital Workplace (FKPDW). Workshop within the 9th Conference on Professional Knowledge Management (M. Leyer, A. Richter, and S. Vodanovich, eds.), pp. 25-40, KIT: Karlsruhe, 2017.

[5] A. H. Bond and L. Gasser, "Readings in distributed artificial intelligence," 1988.

[6] M. Hülsmann and K. Windt, eds., Understanding Autonomous Cooperation and Control in Logistics.
The Impact of Autonomy on Management, Information, Communication and Material Flow. Berlin, Germany: Springer, 2007.

[7] T. W. Sandholm and V. R. Lesser, "Coalitions among computationally bounded agents," Artificial Intelligence, vol. 94, no. 1-2, pp. 99-137, 1997.

[8] S. J. Russell, P. Norvig, and E. Davis, Artificial intelligence: A modern approach. Prentice Hall series in artificial intelligence, Upper Saddle River and NJ: Prentice Hall, 3rd ed ed., 2010.

[9] J. O. Berndt, "Self-organizing Logistics Process Control: An Agent-based Approach," in Agents and Artificial Intelligence. Third International Conference, ICAART 2011. Revised Selected Papers (J. Filipe and A. Fred, eds.), vol. 271 of Communications in Computer and Informations Science, (Berlin, Germany), pp. 397-412, Springer, 2013.

[10] J. Ferber, Multi-agent systems: an introduction to distributed artificial intelligence, vol. 1. Addison-Wesley Reading, 1999.

[11] H.-J. Bürckert, K. Fischer, and G. Vierke, "Holonic Transport Scheduling with TELETRUCK," Applied Artificial Intelligence, vol. 14, no. 7, pp. 697-725, 2000.

[12] K. Fischer, "Agent-based design of holonic manufacturing systems," Robotics and Autonomous Systems, vol. 27, no. 1-2, pp. 3-13, 1999.

[13] A. Karageorgos, N. Mehandjiev, G. Weichhart, and A. Hämmerle, "Agent-based optimisation of logistics and production planning," Engineering Applications of Artificial Intelligence, vol. 16, no. 4, pp. 335-348, 2003.

[14] S. S. Walker, R. W. Brennan, and D. H. Norrie, "Holonic Job Shop Scheduling Using a Multiagent System," IEEE Intelligent Systems, vol. 20, no. 1, pp. 50-57, 2005.

[15] H. Salima, D. Giovanna, K. Anthony, and C. Cristiano, "On self-organising mechanisms from social, business and economic domains', Informatica, vol. 30, no. 1, pp. 63-71, 2006.

[16] C. Castelfranchi, "Formalising the informal?: Dynamic social order, bottom-up social control, and spontaneous normative relations," Journal of Applied Logic, vol. 1, no. 1, pp. $47-92,2003$.

[17] R. Schumann, A. D. Lattner, and I. J. Timm, "Management-by-exception-a modern approach to managing self-organizing systems," Communications of SIWN, vol. 4, pp. 168-172, 2008.

[18] R. Leitner, F. Meizer, M. Prochazka, and W. Sihn, "Structural concepts for horizontal cooperation to increase efficiency in logistics," CIRP Journal of Manufacturing Science and Technology, vol. 4, no. 3, pp. 332 - 337, 2011. Production Networks Sustainability.

[19] J. O. Berndt, Self-Organizing Multiagent Negotiations. Cooperation and Competition of Concurrently Acting Agents with Limited Knowledge, vol. 344 of DISKI. Berlin, Amsterdam: AKA, IOS Press, 2018.

[20] I. J. Timm, T. Scholz, and O. Herzog, "Emerging capabilities in intelligent agents for flexible production control," Advanced Engineering Informatics Journal. Special Issue on Emergent Synthesis, vol. 20, no. 3, pp. 247-259, 2006.

[21] M. L. Moore, R. B. Reyns, S. R. T. Kumara, and J. R. Hummel, "Distributed intelligent agents for logistics (dial)," in 1997 IEEE International Conference 
on Systems, Man, and Cybernetics. Computational Cybernetics and Simulation, vol. 3, pp. 2782-2787 vol.3, Oct 1997.

[22] P. Davidsson, L. Henesey, L. Ramstedt, J. Törnquist, and F. Wernstedt, "An analysis of agent-based approaches to transport logistics," Transportation Research Part C: Emerging Technologies, vol. 13, no. 4, pp. 255 - 271, 2005. Agents in Traffic and Transportation: Exploring Autonomy in Logistics, Management, Simulation, and Cooperative Driving.

[23] C. Bierwirth, Adaptive search and the management of logistic systems: Base models for learning agents, vol. 11. springer science \& Business media, 2012.

[24] J. Wojtusiak, T. Warden, and O. Herzog, "Machine learning in agent-based stochastic simulation: Inferential theory and evaluation in transportation logistics," Computers \& Mathematics with Applications, vol. 64 no. 12, pp. $3658-3665,2012$. Theory and Practice of Stochastic Modeling.

[25] A. I. Schein and L. H. Ungar, "Active learning for logistic regression: an evaluation," Machine Learning, vol. 68, pp. 235-265, Oct 2007.

[26] V. B. Hinsz, R. S. Tindale, and D. A. Vollrath, "The emerging conceptualization of groups as information processors," Psychological bulletin, vol. 121, no. 1, pp. 43-64, 1997.

[27] S. W. J. Kozlowski and D. R. Ilgen, "Enhancing the effectiveness of work groups and teams," Psychological Science in the Public Interest, vol. 7, no. 3, pp. 77-124, 2006.

[28] T. Ellwart, U. Konradt, and O. Rack, "Team mental models of expertise location: Validation of a field survey measure," Small Group Research, vol. 45, no. 2, pp. 119-153, 2014.

[29] E. Salas, S. M. Fiore, and M. P. Letsky, eds., Theories of team cognition - Cross-disciplinary perspectives. New York: Taylor \& Francis Group, 2012.

[30] T. Ellwart and C. H. Antoni, "Shared and distributed team cognition and information overload. evidence and approaches for team adaptation.," in Information and Communication Overload in the Digital Age, IGI Global, 2017 in press.

[31] A. Wiedow, U. Konradt, T. Ellwart, and C. Steenfatt, "Direct and indirect effects of team learning on team outcomes: A multiple mediator analysis," Group Dynamics: Theory, Research, and Practice, vol. 17, no. 4, pp. 232-251, 2013.

[32] R. Oertel and C. H. Antoni, "Phase-specific relationships between team learning processes and transactive memory development," European Journal of Work and Organizational Psychology, vol. 24, no. 5, pp. 726-741, 2015.

[33] T. Ellwart, C. Happ, A. Gurtner, and O. Rack, "Managing information overload in virtual teams: Effects of a structured online team adaptation on cognition and performance," European Journal of Work and Organizational Psychology, vol. 24, no. 5, pp. 812-826, 2015.

[34] M. J. Eppler and J. Mengis, "The concept of information overload: A review of literature from organization science, accounting, marketing, mis, and related disciplines," The Information Society, vol. 20, pp. 325-344, 2004.
[35] A. B. Hollingshead, "Communication, learning, and retrieval in transactive memory systems," Journal of Experimental Social Psychology, vol. 34, pp. 423-442, 1998.

[36] A. B. Hollingshead, N. Gupta, K. Yoon, and D. P. Brandon, "Transactive memory theory and teams: Past, present and future," in Theories of team cognition: Cross-disciplinary perspectives (E. Salas, S. M. Fiore, and M. P. Letsky, eds.), pp. 421-455, New York: Routledge, 2012.

[37] D. M. Wegner, "Transactive memory: a contemporary analysis of the group mind," in Theories of group behavior (B. Mullen and G. R. Goethals, eds.), pp. 185-208, New York: Springer, 1987.

[38] Y. Ren, K. M. Carley, and L. Argote, "The contingent effects of transactive memory: When is it more beneficial to know what others know?," Management Science, vol. 52, no. 5, pp. 671-682, 2006.

[39] R. D. Rao and L. Argote, "Organizational learning and forgetting: The effects of turnover and structure," European Management Review, vol. 3, no. 2, pp. 77-85, 2006.

[40] S. Rao and M. P. Georgeff, "Bdi agents: From theory to practice," in Proceedings of the First International Conference on MultiAgent Systems (ICMAS 1995) (V. R. Lesser and L. Gasser, eds.), (Boston), pp. 312-319, The MIT Press, 1995.

[41] M. Wooldridge, Reasoning about Rational Agents. Cambridge: The MIT Press, 2000.

[42] M. Wooldridge and A. Lomuscio, "Multi-agent VSK logic," in European Workshop on Logics in Artificial Intelligence, pp. 300-312, Springer, 2000.

[43] P. Nyhuis and H.-P. Wiendahl, Fundamentals of production logistics: theory, tools and applications. Springer Science \& Business Media, 2008.

[44] B. Klefsjö, H. Wiklund, and R. L. Edgeman, "Six sigma seen as a methodology for total quality management," Measuring business excellence, vol. 5, no. 1, pp. 31-35, 2001.

[45] M. M. Omodei and A. J. Wearing, "The fire chief microworld generating program: An illustration of computer-simulated microworlds as an experimental paradigm for studying complex decision-making behavior," Behavior Research Methods, Instruments, \& Computers, vol. 27, pp. 303-316, Sep 1995.

[46] L. Reuter, J. O. Berndt, and I. J. Timm, "Challenges of simulating teamwork in organizational scenarios," in 2017 Winter Simulation Conference (WSC), pp. 4542-4543, Dec 2017.

[47] I. J. Timm and F. Lorig, "A survey on methodological aspects of computer simulation as research technique," in Proceedings of the 2015 Winter Simulation Conference, Huntington Beach, CA, USA, December 6-9, 2015, pp. 2704-2715, 2015.

[48] C. M. MacLeod, "Half a century of research on the stroop effect: an integrative review.," Psychological bulletin, vol. 109, no. 2, p. 163, 1991.

[49] M. R. Endsley, "Toward a theory of situation awareness in dynamic systems," Human factors, vol. 37, no. 1, pp. 32-64, 1995. 